\title{
Application of Stepwise Small Dural Window and External Intraventricular Drainage for Craniectomy in Patients with Severe Brain Injury
}

Z Zhijie, W Zhenzhong, Z Bo, C Liqun

\begin{abstract}
Objective: The present study was designed to discuss the practicability and value of stepwise small dural window and Paine's point puncture mediated external intraventricular drainage (EID) for craniectomy in patients with severe brain injury compared with standard trauma craniectomy.
\end{abstract}

Methods: Ninety patients with an Glasgow Coma Scale score less than 8 or less were randomly divided into two groups: standard trauma craniectomy group (44 patients, control group) and stepwise small dural window and Paine 's point puncture mediated EID group (46 patients, improved group) and the clinical data were compared.

Results: The improved group had no statistically significance with control group on duration of surgery and the incidence of intracranial infection $(\mathrm{p}=0.732,0.774)$. However, the improved group had statistically significance with control group on the incidence of intraoperative encephalocele, postoperative delayed hematoma, postoperative epilepsy and hydrocephalus in need of extra surgery $(P=0.007 、 0.020 、 0.014 、 0.011)$. Glasgow outcome score ( GOS ) comparison half a year later showed that the control group had 17 cases of good prognosis, while the improved group had 29 cases, which had statistically significance $(P<0.05)$.

Conclusion: Improved method proved to be an excellent technique on hematoma evacuation in craniectomy in patients with severe brain injury, especially in the prevention and treatment of intraoperative brain swelling, clearance of bloody cerebrospinal fluid and continuously reduce the intracranial pressure. A positive neurological outcome and decreased mortality was also observed in the improved group.

Keywords: Craniectomy, EID, improved stepwise small dural window, severe brain injury

From: Department of Neurosurgery, The affiliated Yangming Hospital of Ningbo University, Yuyao315400,

Zhejiang, China.

Correspondence: Dr Chen Liqun, Department of Neurosurgery, The Affiliated Yangming

Hospital of Ningbo University, Yuyao, Zhejiang, China, E-mail: $925403713 @ q q . c o m$ 


\section{INTRODUCTION}

Acute craniocerebral trauma is a severe clinical condition that commonly occurs in trauma patients. Brain edema and swelling often occur during traumatic brain injuries. Severe traumatic brain injury (STBI), defined as a head trauma that is associated with a Glasgow Coma Scale (GCS) score of 3-8, leads to increased mortality and morbidity and poses a major problem in critical care medicine with poor prognosis. STBI leads to extensive brain tissue contusion and secondary brain injury, which is characterized by brain tissue volume expansion and severe cerebral edema that ultimately results in intractable intracranial hypertension. In recent years, although the great progress has been made in clinical diagnosis and treatment and related basic research to traumatic brain injury, the mortality and morbidity of it still remain the highest (1). And decompressive craniectomy (DC) is still the main and effective treatment for patients with acute severe craniocerebral injury as it is easy to carry out (2). DC is a surgical procedure in which part of the skull is removed to allow the brain to swell without being squeezed. DC can prevent the increase in cranial volume and reduce ICP, thereby preventing the occurrence of cerebral hernia and restoring the cerebral blood flow perfusion. However, more and more surgery complications such as subdural effusion, hydrocephalus, infection, incision incarcerated, epilepsy, intraoperative concurrent hemorrhage, acute encephalocele and early postoperative delayed intracranial hematoma (3-6), and so on occur in the conventional methods, which usually cause deadly consequences. In order to reduce or avoid these complications, many clinicians put forward many improved methods, such as decompressive craniectomy with lattice duraplasty (7) and ladder intracranial pressure-reducing by intracranial pressure monitoring (8). But, the overall 
effects are not ideal (9). So how to reduce the complications of the patients with acute severe craniocerebral injury are still the main problems in the clinical practice.

To reduce intracranial pressure fast and smoothly, at the same time to reduce the complications such as encephalocele during surgery and mortality and morbidity, 90 cases admitted from February 2011 to January 2015 were randomly selected and two methods of acute subdural hematoma removal and bone flap removal for decompression were carried out. During the surgery, we used modified stepwise small dural window and Paine's point puncture mediated external intraventricular drainage (EID). Compared with the conventional surgery, the improved method obtains better effects.

\section{Methods}

\section{Research participants}

A total of 90 cases included in the research including 57 cases of male, 33 cases of female (Table 1). They had an average of $42.6 \pm 6.8$ years of age $(16 \sim 75$ age $)$ and an average of $3.8 \pm 0.5$ hours $(1 \sim 24$ hours $)$ of surgery. Their states were diagnosed by a neurosurgeon physician in the affiliated Yangming Hospital of Ningbo University. Participants were included according to 1) application of craniotomy for hematoma evacuation and decompression within 24 hours after admission; 2) a clear history of head injury with emergency surgical indications by skull CT scanning including one or unilateral intracranial hematoma, midline shift, compression of basal cistern; 3) Glasgow Coma Scale $(\mathrm{GCS}) \leq 8$ before surgery. 
Table 1: Characteristics of all subjects

\begin{tabular}{lll}
\hline & Control group $(\mathbf{n}=\mathbf{4 4})$ & Improved group $(\mathbf{n}=\mathbf{4 6})$ \\
\hline Male & 29 & 28 \\
Female & 15 & 18 \\
Age & $42.5 \pm 6.72$ & $41.2 \pm 5.4$ \\
Average time after injury (h) & $3.7 \pm 0.6$ & $3.9 \pm 0.8$ \\
GCS score & $6.4 \pm 0.85$ & $6.1 \pm 0.7$ \\
\hline
\end{tabular}

There had no statistical significance between two groups $(P>0.05)$.

Among all participants, GCS of 17 cases were $7 \sim 8,41$ cases were $5 \sim 6$ and 32 cases were 3

4. 58 cases had unilateral pupil dilation and 32 cases with bilateral pupil dilation. The exclusion criteria included that 1) patients had severely abnormal preoperative coagulation function or took anticoagulation and antiplatelet drugs; patients needed surgical treatment for intercurrent severe fractures (such as: pelvic, femoral, spine fracture, and so on.) or the chest injury; patients did cerebral surgery within 2 weeks; patients needed ventilator support or (and) vascular active drugs because of respiratory arrest or unstable vital signs.

\section{Surgery}

Standard trauma big bone flap craniotomy group (Control group): incision began with $1 \mathrm{~cm}$ before tragus above zygomatic arch, then continued along oblique line above auricle to the 
back around top nodules, and then went to the halfway point of sagittal line and finally forward to the hair line. Bone window covered an area about $12 \times 15 \mathrm{~cm}$ front to skin, down to the zygomatic arch, up $2 \mathrm{~cm}$ away from the midline and the rest close to the skin edge. Removed the lateral sphenoid ridge, one-time cut open epidural and removed intracranial hematoma. After making sure that there was no active bleeding, pave hemostatic gauze on the residual cavity surface. For severe cerebral contusion induced encephalocele, removed deactivated brain tissues and reduced pressure when necessary. Tension-reducing suture by using artificial dura mater and big bone flap decompression were carried out after surgery.

Improved stepwise small dural window+Paine's point puncture mediated EID group (improved group): incision began with $0.5 \mathrm{~cm}$ before tragus above zygomatic arch, then continued along oblique line above the auricle to the front under top nodules, and next turned to front until $2.5 \mathrm{~cm}$ beside the midline, and then turned down to hair line close to the center line. Bone window area was about $9 \times 11 \mathrm{~cm}$. Generally, first cut temporal (or forehead) dura mater no more than $4 \mathrm{~cm}$, then treated lesions and carried out imprecise stitching (or artificial meningeal tension-reducing suture) to about $2 \mathrm{~cm}$, then cut open the frontal or temporal epidural no more than $4 \mathrm{~cm}$, treated lesions and stitched epidural less than $2 \mathrm{~cm}$. Dealt with top or occipital when necessary, and finally opened the sylvius canal to ensure epidural opened during the whole surgery without being stuck or pressed and the operation process controllable. If the brain pressure was not very high during surgery, appropriately extended dural incision for convenient operation. For intraoperative severe cerebral contusion with brain swelling induced encephalocele, removed deactivated brain tissues and reduced pressure when necessary. Ipsilateral Paine's point (or improved Paine's point) was punctured 
for EID. Tension-reducing suture by using artificial dura mater and bone flap decompression were carried out after surgery. Drainage tube connected to one-time craniocerebral external drainage device.

\section{Evaluation of surgical curative effects}

The comparison of the two groups of patients was done on the basis of the duration of surgery, the incidence of brain bulging, postoperative delayed hematoma (need extra surgery), hydrocephalus in need of surgery, postoperative epilepsy and intracranial infection. Prognosis according to GOS was also compared.

\section{Statistical analyses}

Statistical analyses were performed using the SPSS statistical package version 21.0 (SPSS, Chicago, IL, USA). Variables are expressed as mean \pm Standard Deviation (SD). Means were compared by chi-square check or $t$ test. The level of statistical significance was set at 0.05 .

\section{RESULTS}

\section{Comparison of surgery-related information in the short term}

Comparison of operation-related information in the short term (Table 2) showed that there was no statistically significant between the two groups on the duration of surgery, and incidence of intracranial infection $(P>0.05)$. However, there was statistically significant on the incidence of intraoperative brain bulging, postoperative hematoma, hydrocephalus in need 
of surgery and postoperative epilepsy after half a year $(\mathrm{P}<0.05)$.

Table 2: Comparison of operation-related information in the short term

\begin{tabular}{lllll}
\hline Group & Control group & Improved group & chi-square or & $\boldsymbol{P}$ \\
& $(\mathbf{n = 4 4 )}$ & $(\mathbf{n = 4 6 )}$ & $\boldsymbol{t}$ values & 0.628 \\
\hline Duration of surgery (h) & $2.45 \pm 0.62$ & $2.16 \pm 0.55$ & 0.305 & 0.007 \\
Intraoperative brain bulging & $9(20.5 \%)$ & $1(4.3 \%)$ & 7.525 & 0.020 \\
Late hematoma & $11(25.0 \%)$ & $3(6.5 \%)$ & 5.846 & 0.964 \\
Intracranial infection & $2(4.5 \%)$ & $2(4.35 \%)$ & 0.02 & 0.011 \\
Hydrocephalus & $12(27.3 \%)$ & $3(6.5 \%)$ & 6.972 & 0.014 \\
\hline
\end{tabular}

\section{GOS score comparison half a year later}

The results showed that control group included 17 cases of good prognosis (good and moderate disability) and 27 cases of poor prognosis (severe disability, vegetative survival and death).The improved group showed 29 cases of good prognosis and 17 cases of poor prognosis. There was statistically significant between the two groups $(\mathrm{P}=0.031, P<0.05)$. 
Table 3: Comparison of GOS score after half a year

\begin{tabular}{lcc}
\hline \multicolumn{1}{c}{ Control group } & Improved group \\
& $(\mathbf{n = 4 4 )}$ & $(\mathbf{n}=\mathbf{4 6})$ \\
\hline Good & 11 & 15 \\
Moderate disability & 6 & 14 \\
Severe disability & 7 & 7 \\
vegetative survival & 6 & 4 \\
Death & 14 & 6 \\
\hline
\end{tabular}

\section{DISCUSSION}

In present study, an improved craniectomy received a better effect. The reasons are mainly because:

Standard decompressive craniectomy, due to its large bone flap covering frontotemporal top, can remove $95 \%$ of unilateral acute intracranial hematoma. Our improved method avoid the wound around top nodules, because top occipital brain damages are very rarely and also due to anatomical relationship, the vast majority of cerebral contusions locate at the frontal and sphenoid ridge sides. If top occipital hematoma does occur which are usually postoperative epidural hematoma, we can open nearest bone hole to remove the hematoma, which can increase the natural protection to the large craniocerebral wounds by 
Bone Bridge and reduce postoperative related complications such as external pressure and brain tissue traction because of too large skull defect.

The application of improved craniectomy at the top and frontoposterior position about $2.5 \mathrm{~cm}$ from the center line can avoid the nearby arachnoid granulations and greatly reduce the blood loss of bone flap.

For traditional craniotomy, incision is done $1.0 \mathrm{~cm}$ from tragus above zygomatic arch, but here it is the mainstream of superficial temporal artery which is easy to hurt during the cut of the temporalis, cranial closure suture and second incision and suture of a skull repair. Whereas the improved incision is $0.5 \mathrm{~cm}$ from there, which can effectively avoid the temporal artery, improve the skin flap blood supply, reduce the incidence of infection and reserved for possible anatomic terms for intracranial vascular anastomosis. There were 10 cases of patients with incision infection ( 8 cases in normal group and 2 cases in improved group). They were ischemia, split, fester, and even necrosis caused by oppression of elastic bandage, for the bone window edge was too close to skin incision. 4 cases of infections extended to intracranial and was relieved after repeated treatment with large dose of antibiotics.

By application of improved small dural window, epidural is cut step by step with every part less than $4 \mathrm{~cm}$ in length and imprecise surgical stitching. After treatment of one part of brain contusion or hematoma and then deal with another part. If reduction of intracranial pressure are not satisfied, we can return to dismantle the suture to do epidural tension reduction and even intracranial decompression, and then carefully stitch epidural. Multiple wounds can be handled with the same method. If less severe cerebral contusion, 
epidural is cut with tension-reducing suture at the same time to make brain tissue remain in the epidural cavity and make the changes of intracranial pressure in a state of control. In this way, dura mater can play a role of natural protector to avoid the large leakage of brain tissues during surgery, so reduce intracranial pressure slowly and evenly and greatly reduce brain tissue incarceration, late bleeding, intraoperative acute encephalocele, and sudden drop of blood pressure, which completely avoid intraoperative encephalocele that cranial cannot be closed. There were 10 patients with encephalocele including 9 cases in normal group and 1 in improved group. At the same time, inadequate decompression, difficult treatments to trauma and inner decompression because of too small incision and cerebrospinal fluid leakage induced by epidural incompletely suture won't occur like epidural mesh cut surgery.

Paine's point (or improved Paine's point) (10) is often used in ventricle puncture of pterion approach craniotomy. In recent years, Paine's puncture is used in aneurysm surgery to reduce intracranial pressure which obtain good curative effect. Furthermore Paine's point puncture can be made within the same wounds and don't need to adjust the position and do another cut during surgery, so reduce damage and shorten the surgery time.

Ventricular drainage is the first aid measure for increasing pressure which is usually used in hydrocephalus, intraventricular hemorrhage and central nervous system infection diseases. In recent years, some clinicians used it to treat craniocerebral trauma and received certain effects (11). The EID quickly carry hemorrhagic cerebrospinal fluid out to reduce intracranial pressure and cerebral inner pressure. Swelling and edema of brain tissues cause high pressure and pressure gradient, which prompted edema fluid flow towards the ventricle where pressure is low that block the occurrence and development of traumatic acute diffuse 
brain swelling and reduce the use of dehydration drug. Continuous drainage can clear off all kinds of active substances secreted by breakdown of red blood cells which lead to cerebral vasospasm and acute inflammation, thereby effectively avoid cerebral vasospasm and fatal complications such as traumatic cerebral infarction and severe inflammation reaction. However, cerebral ventricle squeezed smaller and was difficult to puncture successfully in severe craniocerebral injury with increased intracranial pressure. Long detaining of tube easily cause secondary fatal intracranial infection. Our approach is that the catheter is placed after removal of focus and closed temporarily after confirmation of the success of the catheter. Intermittently open the catheter according to the condition of intracranial extubation after surgery. Extubate in 5 days as far as possible. If intracranial pressure remains high after 7 days, the contralateral frontal drainage or lumbar puncture was done again when necessary. The patients in present study exported cerebrospinal fluid $20+320 \mathrm{ml}$ (average $120+20 \mathrm{ml}$ ) every day with catheter time $1 \sim 7$ days (average of $3.3 \pm 0.6$ days).

EID can reduce the area of bone window induced by improved bone flap. Craniocerebral external drainage device (completely closed) can reduce the cost and indirectly monitor intracranial pressure, which provide the basis for postoperative treatment and prognosis assessment.

Emergency surgery for craniocerebral injury is often urgent without much time to prepare. Intraoperative application of small dural window strategy dealing with deep lesions requires good skills which means the clinicians are familiar with anatomy to avoid failure of the puncture and fall of drainage tube. Being ensuring that there is no encephalocele, dural incision can moderately lengthen to help stop bleeding in the deep and improve efficiency. 
Through the application of improved DC, we have received a better therapeutic effect. However, surgery is only one of the key factors of prognosis. The opportunity of surgery, respiratory tract management, blood pressure, water and electrolyte balance, blood coagulation dysfunction etc., all have direct impact on treatments. So, new methods and principles need to be constantly improved for treatment of severe head injury. 


\section{REFERENCES}

1. Joseph B, Haider A, Rhee P. Traumatic brain injury advancements. Curr Opin Crit Care 2015; 21: 506-11.

2. Vital RB, Hamamoto Filho PT, Ducati LG, Braga GP, Nunes HR et al. Decompressive Hemicraniectomy in a South American Population - Morbidity and Outcomes Analysis. PLoS One 2016; 11: e0146747.

3. Brommeland T, Rydning PN, Pripp AH, Helseth E. Cranioplasty complications and risk factors associated with bone flap resorption. Scand J Trauma Resusc Emerg Med 2015; 23: 75.

4. Grille P, Tommasino N. Decompressive craniectomy in severe traumatic brain injury: prognostic factors andcomplications. Rev Bras Ter Intensiva 2015; 27: $113-8$.

5. Liang ES, Tipper G, Hunt L, Gan PY. Cranioplasty outcomes and associated complications: A single-centre observational study. Br J Neurosurg 2015; 2:1-6.

6. Kurland DB, Khaladj-Ghom A, Stokum JA, Carusillo B, Karimy JK, Gerzanich V et al. Complications Associated with Decompressive Craniectomy: A Systematic Review. Neurocrit Care 2015; 23: 292-304.

7. Mitchell PI, Tseng M, Mendelow AD. Decompressive craniectomy with lattice duraplasty. Acta Neurochir 2004; 146: 159-60.

8. Kawoos U, McCarron RM, Auker CR. Advances in Intracranial Pressure Monitoring and Its Significance in Managing Traumatic Brain Injury. Int J Mol Sci 2015; 16: 28979-97. 
9. Tagliaferri F, Zani G, Iaccarino C. Decompressive craniectomies, facts and fiction: a retrospective analysis of 526 cases. Acta Neurochir (Wien) 2012; 154: 919-26

10. Park Jaechan, Hamm In-Suk. Revision of Paine's technique for intraoperative ventricular puncture. Surg Neurol 2008; 70: 503-8.

11. Bhargava D, Alalade A, Ellamushi H, Yeh J, Hunter R. Mitigating effects of external ventricular drain usage in the management of severe head injury. Acta Neurochir 2013; 155: 2129-32. 